Baden-Badener Strafrechtsgespräche

Fischer/Hoven/Huber/Raum/Rönnau/Saliger/Trüg (Hrsg.)

Dogmatik und Praxis des strafrechtlichen Vermögensschadens 


\section{Vermögensschaden bei Personengesellschaften \\ - Zur Normativierung des Vermögensbegriffs -}

Michael Kubiciel

\section{Einheit der Bezeichnung - Spaltung des Begriffs}

Einen eigenständigen und in seinem Umfang zumindest bestimmbaren Anwendungsbereich weist der Betrugstatbestand nur auf, wenn die deliktstypische Handlung (die Täuschung) und der tatbestandsmäßige Erfolg (der Vermögensschaden) einigermaßen klar konturiert und widerspruchsfrei konzeptualisiert werden können. Die Rechtsanwender können heute zwar auf eingängige Definitionen der Täuschung und des Vermögensschadens zurückgreifen. Jedoch vermögen diese kaum zu verdecken, dass die zentralen Begriffe Täuschung, Vermögen und Vermögensschaden ungeklärt sind. Rechtspraxis und Rechtswissenschaft versuchen jedenfalls nicht selten, Gegensätzliches unter ein- und dieselbe Bezeichnung zu fassen. Dies gilt auch für den Tatbestand der Untreue, namentlich das tatbestandsmäßige Verhalten und den Begriff des Vermögensnachteils i.S. des $\$ 266$ StGB.

\section{Normativierungen des faktischen Täuschungsbegriffs}

So will die h.M. eine ausdrückliche Täuschung mit Hilfe einer faktischen Betrachtungsweise feststellen, ${ }^{1}$ obgleich sie bei einer konkludenten Täuschung auf normative Erwägungen zurückgreifen muss. ${ }^{2}$ Ausschlagge-

1 Vgl. Cramer/Perron, in: Schönke/Schröder, StGB, 29. Aufl. 2014, §263 Rn. 13; Hoyer, in: SK-StGB, Bd. 4, 7. Aufl., 60. Lfg., Februar 2004. § 263 Rn. 18; Tiedemann, in: LK-StGB, Bd. 9/1, 12. Aufl. 2012, § 263 Rn. 25; Wittig, Das tatbestandsmäßige Verhalten des Betruges, 2005, S. 382 ff.; s. auch Satzger, in: Satzger/ Schluckebier/Widmaier, StGB. 2. Aufl., 2014, § 263 Rn. 29, 36.

2 Zur konkludenten Täuschung Frisch, Festschrift Jakobs. 2007, S. 97 ff.; Kasiske, GA 2009, 360 ff.; Kindhäuser, Festschrift Tiedemann, 2008, S. 581 ff.; Kubiciel, HRRS 2007, 68 ff.; ders., JZ 2010, 422, 423; Vogel, in: Gedächtnisschrift Keller, 2003, S. $313 \mathrm{ff}$. 
bend sind normative Erwägungen auch in den Fällen, in denen bspw. Verkäufer Wissens- und Informationsvorsprünge planmäßig ausnutzen, ohne dass dies für strafwürdig und tatbestandsmäßig erachtet wird. ${ }^{3}$ Anders als die Definition der h.M. Glauben macht, ist also keineswegs alles Beweisbare eine Tatsache und nicht jede Lüge stellt eine Täuschung dar. Damit geht die Einheit des Täuschungsbegriffs verloren: Es wird bald mit einem ontologischen, bald mit einem normativen Begriff operiert. ${ }^{4}$ Diese Begriffsspaltung ist alles andere als ein peripheres Problem, sondern betrifft den Kern des Handlungsunrechts und damit einen von zwei zentralen Elementen des Betrugstatbestandes.

\section{Der Vermögensschaden zwischen ökonomischer Begriffsbildung und normativer Korrektur}

Auch auf der Erfolgsseite des Betruges lässt sich eine rechtspraktische und rechtsdogmatische Unsicherheit beobachten, die Konsequenz einer heterogenen Begriffsbildung ist. Folgen hat diese Heterogenität nicht nur für den Betrugs-, sondern auch für den Untreuetatbestand. Zuletzt deutlich geworden ist dies an dem Streit um die sog. schadensgleiche Vermögensgefährdung $^{5}$ oder anders gewendet: den Gefährdungsschaden ${ }^{6}$. BVerfG und h.L. begegnen dieser Figur mit deutlichen Vorbehalten. Dabei ist sie lediglich eine konsequente Umsetzung des „,bewährten“7 Vermögensbegriffs, der ökonomisch fundiert und nur auf zweiter Ebene ,juristisch korrigiert"

3 Heinrich, in: ArztWeber/Heinrich/Hilgendorf, Strafrecht Besonderer Teil, 3. Aufl. 2015, § $20 \mathrm{Rn} .7 \mathrm{ff}$.

4 Anders die im Vordringen begriffenen normativierenden Konzeptionen, s. dazu Frisch, Festschrift Herzberg, 2008, S. 729, 738 ff.; Jakobs, Festschrift Tiedemann, 2008, S. 649, 654 f.; Kindhäuser, in: Kindhäuser/Neumann/Paeffgen, Nomos Kommentar zum Strafgesetzbuch, 3. Bd., 4. Aufl. 2013, § 263 Rn. 63 ff.; dens., ZStW 103 (1991), 398 ff.; dens., Festschrift Bemmann, 1997, S. 339 ff.; Kubiciel, HRRS 2007, 68, 69 ff.; dens., JZ 2010, 422, 423; Pastor Muñoz, GA 2005, 129, 133; Pawlik, Das unerlaubte Verhalten beim Betrug, 1999, S. 82, 93 ff., 193 ff.; dens., Festschrift für Lampe, 2003, S. $689 \mathrm{ff}$; $T$. Walter, Betrugsstrafrecht in Frankreich und Deutschland, 1999, S. 72. - Krit. Vogel (Fn. 2), S. 318 ff.: ebenfalls kritisch, trotz eines ,auch normativen Täuschungsbegriffs“ Saliger, in: Matt/Renzikowski, StGB, 2013, § 263 Rn. 27.

5 Lenckner, JZ 1971, 320, 323.

6 Kindhäuser (Fn. 4). \$263 Rn. 297.

7 Wessels/Hillenkamp, Strafrecht BT 2, 37. Aufl. 2014, Rn. 534. 
wird. Da sich der Wert einer Sache oder einer Forderung in einem marktwirtschaftlichen System dynamisch, d.h. mit dem Verhältnis von Angebot und diesbezüglichen Erwartungen der Marktteilnehmer entwickelt, ${ }^{8}$ mindert die reale Gefahr, dass eine Sache nicht zurückerlangt werden kann oder als Forderung (teilweise) ausfallen wird, den ökonomischen Tauschwert. ${ }^{9}$ Das Resultat ist keine banale „Belästigung"10 oder „bloße“ Gefährdung des Vermögens, ${ }^{11}$ sondern eine reale ökonomische Werteinbuße. ${ }^{12}$ Die Annahme eines Gefährdungsschadens ist mithin eine schlichte Anerkennung ökonomischer Tatsachen, kein Resultat einer (unzulässigen) Normativierung. ${ }^{13}$ So betrachtet, liegen auch nicht die Voraussetzungen im Dunkeln, unter denen eine Gefährdungslage als schadensgleich zu bewerten ist. ${ }^{14}$ Unklar ist vielmehr, aus welchen Gründen und unter welchen Voraussetzungen ein ökonomisch-empirisch nachweisbarer (Gefährdungs-)Schaden als nicht schadensgleich bewertet werden soll. ${ }^{15} \mathrm{Nicht}$ die Begründung der Schadensqualität einer Vermögensgefährdung, sondern die wertende Aussonderung dieser Schadensart aus dem Tatbestand ist eine Normativierung. Die Weite des herrschend vertretenen, primär ökonomischen Vermögensbegriffs wird also nicht zum Anlass genommen, diesen kritisch zu hinterfragen; vielmehr werden seine Konsequenzen mit Hilfe von systemfremden (strafrechtsdogmatischen, kriminalpolitischen oder verfassungsrechtlichen) Erwägungen zurückgeschnitten. Infolgedessen kann der Anwender des Betrugstatbestandes nicht auf einen klar konturierten Begriff des Vermögensschadens zurückgreifen, sondern muss

8 Vgl. zu dieser Dynamik Fischer, NStZ-Sonderheft 2009, 8, 11; Panlik (Fn. 4), S. 153 f.; Otto, Festschrift für Lackner. 1987, S. 715, 724: Wittig (Fn. 1). S. 381.

9 So auch BVerfGE 126, 170, 223; Otto (Fn. 8), S. 724 f.

10 Naucke, StV 1985, 187.

11 In diese Richtung aber Beulke/Witzigmann, JR 2008, 430, 433.

12 BGHSt 53, 199, 202 f.; Becker. HRRS 2009, 334, 336; Fischer, StraFo 2008, 269, 271; ders., StV 2010, 95, 97 f.; ders., StGB, 62. Aufl. 2015, § 263 Rn. 157; Lenckner, JZ 1971, 320.321; Mitsch, Strafrecht Besonderer Teil, Bd. 2/1, 2. Aufl. 2003, $\S 7$ Rn. 97; Rengier, Strafrecht Besonderer Teil, Bd. 1, 17. Aufl. 2015, §13 Rn. 184 f.; Satzger, Jura 2009, 518, 519; Tiedemann (Fn. 1), \$263 Rn. 168. S. aber Schünemann, NStZ 2008, 430. 432.

13 Vgl. Saliger, Festschrift Samson, 2010, S. 455, 456, 467; dens., ZIS 2011, 902, 912, einerseits; Fischer, StV 2010, 95, 98 andererseits.

14 So aber Dierlamm, in: Münchener Kommentar zum StGB, Bd. 5, 2014. § 266 Rn. 211.

15 Vgl. Tenckhoff, Festschrift für Lackner, 1987, S. 677, 679 („normativen Begrenzung des Schadensbegriffs"). 
versuchen, unterschiedlichen, teils gegenläufigen ökonomischen straf,verfassungs- und bilanzrechtlichen Vorgaben gerecht zu werden. ${ }^{16}$ Fehlt ein übergeordnetes (systematisches) Prinzip, können Konflikte zwischen unterschiedlichen (ökonomischen, rechtlichen) Topoi lediglich im Wege einer Ad-hoc-Begründung aufgelöst werden, welche die Kasuistik um immer weitere Fallgruppen erweitern, während sie eine die Vorhersehbarkeit und Gleichmäßigkeit der Rechtsanwendung ermöglichende Systematisierung weiter erschweren.

Die Kasuistik folgt letztlich aus der „Prinzipienlosigkeit“ des heterogenen, weil ökonomische mit normativen Aspekten vermengenden Vermögensbegriffs der herrschenden Meinung. ${ }^{17}$ Auch eine andere, stark diskutierte Fragestellung speist sich aus dieser zwischen faktisch-ökonomischen und normativen Aspekten changierenden Modellierung und Anwendung des Vermögensbegriffs. Zweifelhaft ist hier nicht, ob ein Schaden entstanden ist, sondern wo bzw. bei wem er zu lokalisieren ist. Konkret gesprochen, lautet die Frage: Können (auch) Personengesellschaften einen tatbestandsmäßigen Vermögensschaden erleiden oder (auch bzw. nur) die hinter diesen stehenden (natürlichen oder juristischen) Personen? ${ }^{18}$ Dieser Frage soll im Folgenden im Lichte des strafrechtlichen Vermögensbegriffes nachgegangen werden.

16 Zutr. kritisch Fischer (in diesem Band), S. 51 ff., insbes. S. 54. S. aber auch Saliger (in diesem Band), S. 15. ff.; Schünemann (in diesem Band), S. 61, 70 f.

17 Kritik an dieser Prinzipienlosigkeit bei Hefendehl, Vermögensgefährdung und Expektanzen, 1994, S. 110 f., 115; Pawlik (Fn. 4), S. 258 f. (Zitat auf S. 259); Nelles, Untreue zum Nachteil von Gesellschaften, 1991, S. 402, 407 f., 412.

18 Umfassend dazu Brand, Untreue und Bankrott in der KG und GmbH \& Co. KG, 2010, S. 35 ff.; Soyka, Untreue zum Nachteil von Personengesellschaften, 2008, S. $21 \mathrm{ff}$., $96 \mathrm{ff}$., $247 \mathrm{ff}$;; Stölting, Das Tatbestandsmerkmal des fremden Vermögens bei der Untreue zum Nachteil von Personengesellschaften am Beispiel der GmbH \& Co. KG, 2010, S. $29 \mathrm{ff}$. Im Anschluss an BGH, 1 StR 532/12 vom 10.7.2013 (abgedruckt: NJW 2013, $3590 \mathrm{ff}$; JZ 2014, $909 \mathrm{ff}$.), Brand, NJW 2013, $3594 \mathrm{ff}$; Golombek, WiJ 2014, 84 ff.; Lindemann/Hehr, NZWiSt 2014, 350 ff.; Schmidt, JZ 2014,878 ff.; Wessing, NZG 2014, 97 ff. 
II. Das Schadensopfer: eine ökonomische Bezugsgröße oder juristische Person?

\section{Praktische und strafrechtsdogmatische Bedeutung des Streits um die Personengesellschaft}

$\mathrm{Ob}$ eine Personengesellschaft, etwa eine Kommanditgesellschaft (KG), Opfer einer Vermögensschädigung im strafrechtlichen Sinne (§§ 263, 266 StGB) sein kann, ist aus mindestens drei Gründen praktisch bedeutsam: zum einen für die Anforderungen an die Berechnung der Schadenshöhe, ${ }^{19}$ zum anderen für die prozessuale Stellung der Betroffenen - Nebenklägereigenschaft oder Zeugenstellung? - und schließlich für die Auswirkungen eines Einverständnisses von (Teil-)Gesellschaftern auf die Tatbestandsmäßigkeit der fraglichen Handlung. ${ }^{20}$ Die Problematik ist aber auch, was wenig Beachtung findet, von strafrechtsdogmatischer Bedeutung. Grundlegend für ihre Beantwortung ist nämlich nicht die zivilrechtliche Einstufung der Personengesellschaft und die sie treffenden handelsrechtlichen Pflichten, ${ }^{21}$ sondern der strafrechtliche Vermögensbegriff: Begreift man das strafrechtliche Vermögen als eine primär ökonomische Größe, sind wirtschaftliche Erwägungen nicht nur für die Beurteilung ausschlaggebend, ob ein Schaden eingetreten und wie hoch dieser ist. Vielmehr ist nach diesen - wirtschaftlichen - Maßstäben zu entscheiden, wo bzw. bei wem ein Schaden eingetreten ist. Ist, wovon die herrschende Meinung ausgeht, der Vermögensschaden ein Verlust ökonomischer Macht, 22 sind geschädigt i.S. der $\S \S 263,266$ StGB all jene, die tatsächlich wirtschaftliche Werte eingebüßt haben. Welchen rechrlichen Status der Geschädigte hat, ist bei dieser Betrachtungsweise unerheblich. Geht man hingegen umgekehrt davon aus, dass Vermögensschäden nur bei Gesellschaften eintreten können, denen das Recht den Status einer juristischen Person zuweist, gibt

19 Beispielhaft die oben Fn. 18 genannte Entscheidung. hier zitiert nach: BGH NJW $2013,3590,3592 \mathrm{f}$.

20 BGH, NJW 3590, 3593; Schünemann, in: Leipziger Kommentar zum Strafgesetzbuch, Bd. 9/1, 12. Aufl. 2012, § 266 Rn. 262.

21 S. aber die Betonung dieses Aspekts durch Brand, NJW 2013, 3594, 3595; Golombek, WiJ 2014, 84. 91 f.; Lindemann/Hehr, NZWiSt 2014, 350. 353 f.: Schmidt. JZ 2014, $878 \mathrm{ff}$.; Wessing, NZG 2014. 97, 98 f.

22 Konzise Kennzeichnung der herrschenden Meinung bei Parulik (Fn. 4), S. 256; Nelles (Fn. 17), S. 363. 
offenkundig eine normative Betrachtungsweise den Ausschlag, nicht die Ökonomie.

\section{Meinungsstand}

Sowohl die Rechtsprechung als auch die herrschende Meinung in der Literatur gehen, wie gesehen, von einem primär ökonomischen Vermögensbegriff aus, dessen Konsequenzen lediglich partiell durch normative Erwägungen - wenn man will: Kriterien zweiter Ordnung - korrigiert werden. Mit Hilfe dieser Kriterien zweiter Ordnung wird etwa versucht, dem Gefăhrdungsschaden den Status als vollgültigen Schaden i.S. der $\$ \$ 263,266$ StGB zu verweigern; zudem werden nichtigen Forderungen oder sittenwidrig erworbenen Vermögensbestandteilen die strafrechtliche Vermögensschutzwürdigkeit abgesprochen. ${ }^{23}$ Trotz seiner Heterogenität weist der herrschende Vermögensbegriff mithin ein immanentes Stufenverhältnis zwischen einer vorrangigen wirtschaftlichen Betrachtungsweise und der nachrangigen Berücksichtigung normativer Aspekte zum Zweck der Einhegung der Konsequenzen der ökonomischen Sichtweise auf. Umso erstaunlicher ist es, dass sowohl die Rechtsprechung als auch die sie kommentierende Literatur die Frage, ob eine Personengesellschaft einen Vermögensnachteil erleiden kann, ausschließlich anhand von juristischen Kriterien erörtern. Wirtschaftliche Gesichtspunkte, die innerhalb des strafrechtlichen Vermögensbegriffes gemeinhin Kriterien erster Ordnung darstellen, spielen demgegenüber keine Rolle.

Deutlich wird dies an der bereits genannten Entscheidung des 1. Strafsenats aus dem Jahr 2013. Gegenstand der Entscheidung war u.a. die Frage, ob für die Höhe des Vermögensnachteils (i.S. des $\$ 266 \mathrm{StGB}$ ) auf das Vermögen der KG oder auf das Vermögen der Gesellschafter der KG abzustellen ist. ${ }^{24}$ Einer - nicht gänzlich ungebrochenen ${ }^{25}$ - Rechtsprechungslinie folgend betont der Senat, dass die Schädigung des Vermögens einer $\mathrm{KG}$,nach der bisherigen Rechtsprechung des $\mathrm{BGH}^{\circ}$ lediglich dann zu einem straftatbestandsmäßigen Vermögensnachteil führen könne, wenn gleichzeitig das Vermögen der Gesellschafter „berührt" werde. Zwar

23 Statt vieler Hefendehl, in: Münchener Kommentar zum Strafgesetzbuch, Bd. 5. 2. Aufl. 2014, § 263 Rn. 468 ff,; Kindhäuser (Fn. 4), §263 Rn. 234.

24 Oben Fn. 18.

25 Dazu Wessing, NZG 2014, 97, 98, mit Verweis auf BGH, NStZ 2004, 205. 
brächte, so der Senat weiter, die Rechts- und zivilprozessuale Parteifähigkeit ,eine Rechtsstellung mit sich, die der Selbstständigkeit einer juristischen Person in weitem Umfang entsprechen mag. Dessen ungeachtet ist die Kommanditgesellschaft - anders als die Kapitalgesellschaften - aber nach der Rechtsprechung des BGH gerade keine juristische Person.“26 Der Senat stützt seine Entscheidung folglich allein auf die von den Zivilsenaten bekräftigte Unterscheidung von Rechtsfähigkeit einerseits und dem Status einer juristischen Person andererseits. Weder wird begründet, weshalb diese Unterscheidung für das Strafrecht bedeutsam sein soll, noch lässt sich das Ergebnis aus der andernorts hervorgehobenen ,grundsätzlich gebotenen wirtschaftlichen Betrachtung " ${ }^{\text {"27 }}$ ableiten: Aus einer ökonomischen Perspektive wäre nämlich danach zu fragen. wo unmittelbar eine Einbuße an wirtschaftlicher Potenz verursacht worden ist. Dies muss nicht der Gesellschafter, sondern kann - fallabhängig - auch die Personengesellschaft sein.

Trotz dieser offenkundigen Begründungslücken argumentieren auch die Kritiker in der Literatur nicht auf dem Boden des herrschenden strafrechtlichen Vermögensbegriffs, sondern ebenfalls rein zivilrechtlich. Vor allem machen sie geltend, die strafrechtliche Rechtsprechung hätte der gesellschaftsrechtlichen Tendenz zur Angleichung rechtsfähiger Personengesellschaften an die juristische Person folgen sollen. ${ }^{28}$ Der beide Gesellschaftsformen unterscheidende Umstand, dass bei Kapitalgesellschaften die Haftung auf das Gesellschaftsvermögen beschränkt ist, gibt tatsächlich kein starkes Argument für die unterschiedliche Behandlung ab. ${ }^{29}$ Denn die Haftungsbegrenzung thematisiert das Verhältnis zwischen der Gesellschaft und ihren Gläubigern und nicht ihren Schädigern. Anders gewendet: Dass bei einer Personengesellschaft die hinter der Gesellschaft stehenden Personen haften, während bei Kapitalgesellschaften der Haftungsdurchgriff in dieser Form nicht möglich ist, speist sich aus Erwägungen zum Ausgleich von Rechtsverkehrs- und Gläubigerschutz auf der einen und dem Gesell-

26 BGH, NJW 2013, 3590, 3593. S. auch Schünemann (Fn. 20), § 266 Rn. 262: „nach traditioneller (zivilrechtlicher, MK) und heute noch im Strafrecht gültiger Auffassung (....)." Ferner Fischer, StGB. 62. Aufl. 2015, § 266 Rn. 113.

27 So (im Rahmen der Erörterungen zum Vermögensnachteil) BGH. NJW 2013, 3590, 3594.

28 Brand, NJW 2013, 3594 f.; Golombek. WiJ 2014, 84, 92; Gunst, BB 2001. 1537, 1539.

29 Golombek, WiJ 2014, 84. 92. So schon Gunst, BB 2001, 1537, 1539. 
schafterschutz auf der anderen Seite. Derartige Erwägungen haben keine Aussagekraft für die Frage der strafrechtlichen Vermögensqualität des $\mathrm{Ge}-$ sellschaftsvermögens. Stärker ins Gewicht fällt hingegen, dass handelsrechtliche Normen wie $\S \S 124$ Abs. 2, 155 Abs. I HGB von der Existenz eigenen Vermögens der Personengesellschaft auszugehen scheinen. ${ }^{30}$ Derartige Normen ließen sich als Indiz dafür heranziehen. dass das Vermögen insgesamt nicht den Gesellschaftern. sondern der rechtsfähigen Gesellschaft zuzurechnen ist. ${ }^{31}$ Ungeachtet dessen bleibt eine zivilrechtliche Statusdifferenz zwischen Rechtsfähigkeit. ${ }^{32}$ die einer KG zukommt. und der Rechtspersönlichkeit, die der KG fehlt. ${ }^{33}$ Auch die im Gesellschaftsrecht herrschende Meinung stellt beides nicht gleich. ${ }^{34}$ Denn Kapitalgesellschaften zeichnet die deutliche Trennung zwischen Gesellschaft und Gesellschaftern als Folge ihrer mit der Eintragung im Handelsregister erlangten Rechtsnatur als juristische Person aus. Der ihnen zugrundeliegende Gesellschaftsvertrag verselbstständigt sich zur Satzung der AG oder $\mathrm{GmbH}$, ist autonom auszulegen und kann durch qualifizierten Mehrheitsbeschluss geändert werden. Der Rückgang der Gesellschafterzahl auf eine Person verändert die Existenz der Gesellschaft nicht; sie kann sogar als Einmann-Gesellschaft gegründet und weitergeführt werden. Personengesellschaften setzen für ihre Existenz hingegen grundsätzlich den Fortbestand des Gesellschaftsvertrags, d.h. das Vorhandensein von mindestens zwei Gesellschaftern voraus. und es gelten der (dispositive) Grundsatz der Einstimmigkeit und der (zwingende) Grundsatz der Selbstorganschaft.

30 Lindemann/Hehr, NZWiSt 2014, 350, 353.

31 Brand (Fn. 18), S. 210 ff; ders., NJW 2013, 3590, 3595; Ceffinato. Legitimation und Grenzen der strafrechtlichen Vertreterhaftung nach $\$ 14$ StGB, 2010, S. 331; Schmidt, JZ 2014, 878, 881 ff.; Golombek, WiJ 2014, 84, 92 f.; Stölting (Fn. 18), S. $98 \mathrm{ff}$.

32 BGHZ 146, $341 \mathrm{ff}$.

33 Dies betont zu Recht auch Wessing, NZG 2014, 97, 98. Dies (wenig überzeugend) zu relativieren versucht $B$ rand, NJW 2013, 3594 f.

34 Dazu und zum Folgenden Grunewald, Gesellschaftsrecht. 9. Aufl. 2014, S. 50 ff.; UlmeriSchäfer. in: Münchener Kommentar zum Bürgerlichen Gesetzbuch, 6. Aufl. 2013, Bd. 5, § 705 Rn. 308 mit weiteren Nachweisen. 


\section{Freiheitstheoretisch-personaler Vermögensbegriff als Fundament der Rechtsprechung und h.L.}

Der Meinungsstreit ist zwar gesellschaftsrechtlich ergiebig, lässt aber die entscheidende Frage offen, weshalb die verbleibenden Status- und Sachunterschiede zwischen Personen- und Kapitalgesellschaften von strafrechtlicher Relevanz sein sollen. Eine Begründung findet sich in einem freiheitstheoretisch fundierten strafrechtlichen Vermögensbegriff. Dieser begreift das Vermögen ebenso wenig wie die anderen vom Strafrecht garantierten Primärrechte (einer Person) nicht als Museumsstücke, die es zum Nutzen des Einzelnen oder aus Gemeinwohlbelangen schlicht zu erhalten gilt. ${ }^{35}$ Vielmehr ist das Vermögen (ebenso wie alle anderen subjektiven Rechte) für das Strafrecht eine Bedingung zur Entfaltung personaler Freiheit. ${ }^{36}$ Als solches wird es legitimerweise strafrechtlich geschützt, weil die im Straftatbestand vorgesehene Beschränkung der Freiheit des Einzelnen gleichsam der Preis für die Ermöglichung realer Freiheit des Einzelnen in einer konkreten Gesellschaft (mit anderen Bürgern) ist. ${ }^{37}$ Personale Freiheit entfalten können aber nicht nur natürliche Personen, sondern auch juristische Personen. Denn anders als Personengesellschaften gestaltet das Recht sie in einer Weise aus, die ihnen vermittels ihrer Organe und im Rahmen ihrer Satzung die Bildung und Umsetzung eines eigenen Willens gestattet (III. 2., am Ende). Folglich ist auch für juristische Personen das Vermögen Mittel zur Verfolgung eigener Zwecke. ${ }^{38}$ Da Personengesellschaften diese Eigenschaften fehlen, mag ihnen in Einzelbereichen Vermögen rechtlich zugewiesen sein; es untersteht aber nicht

35 Gegen ein solch statisches Verständnis bereits Welzel, ZStW 58 (1938), 491, $515 \mathrm{f}$. Zu Welzel umfassend Frisch/Jakobs/Kubiciel/Pawlik/Stuckenberg (Hrsg.), Lebendiges und Totes in Welzels Verbrechenslehre, 2015. Näher zu der Problematik Amelung, ZStW 87 (1975), 132, 141; Frisch, in: Hefendehl/v. Hirsch/Wohlers (Hrsg.), Die Rechtsgutstheorie. 2003, S. 215, $225 \mathrm{ff}$;; Jakobs, Festschrift für Geilen, S. 76 f.; Kubiciel, Die Wissenschaft vom Besonderen Teil des Strafrechts. Ihre Aufgaben, ihre Methoden, 2013, S. 53 ff.; Pawlik, Das Unrecht des Bürgers. 2012, S. $137 \mathrm{ff}$.

36 Ähnlich Pawlik (Fn. 4), S. 259.

37 Näher dazu mit weiteren Nachweisen Kubiciel (Fn. 35), S. 161 ff., 164 ff.

38 So richtig Kindhöuser (Fn. 4), § 266 Rn. 34, gegen Harrwig, GA 1956, 6 ff. 
ihrer Verfügungsmacht ${ }^{39}$ und ist daher, strafrechtlich betrachtet, nicht $i h r$ Vermögen.

\section{Normativierung als Ausweg aus der Grundlagenkrise der Schadensdogmatik}

Wie die eingangs genannten Beispiele zeigen, befindet sich die Dogmatik des Betrugstatbestandes in einer schweren Krise ihrer Grundlagen. ${ }^{40}$ Ihre Ursache hat diese Krise - wie gezeigt - in der Unschärfe der beiden zentralen Begriffe Täuschung und Vermögensschaden. ${ }^{41}$ Ohne klar konturierte Begriffe aber ist eine stringente Auslegung des „Kunstproduktes ${ }^{6 / 42} \mathrm{Be}$ trug nicht möglich. Dies gilt auch für den Untreuetatbestand, da die h.M. das den Telos des Betrugs- und Untreuetatbestandes bezeichnende Wort Vermögen wie ein ,contested concept ${ }^{43}$ verwendet, in dessen Rahmen sie fallabhängig ganz unterschiedliche Gesichtspunkte ökonomischer, zivil-, handels-, bilanz- oder verfassungsrechtlicher Provenienz zur Geltung bringt (s. oben I. 2). Ein derart unsystematisch-topisches Vorgehen kann aus sich heraus nicht begründen, welche ökonomischen, zivil-, handelsoder verfassungsrechtlichen Erwägungen im Einzelfall strafrechtserheblich sein sollen und welche nicht. ${ }^{44}$ Was für den Einzelfall gilt, gilt auch für die Grundlagen: Da sich Recht und Ökonomie im Verhältnis des Andersseins gegenüberstehen, kann es keine einheitliche und widerspruchsfreie Vermögensschadensdogmatik geben, wenn der herrschende Vermögensbegriff wesentlich Ungleiches zu vereinigen und die dadurch entste-

39 Vgl. zu diesem Element des strafrechtlichen Vermögensbegriffs Kindhäuser (Fn. 4), §266 Rn. 35.

40 So Pawlik (Fn. 4), S. 1. Bereits vor einem halben Jahrhundert nahm Wolfgang Naucke (Zur Lehre vom strafbaren Betrug, 1964, S. 216) ein „beständiges Praktizieren einer unsicheren Methode" bei der Auslegung des § $263 \mathrm{StGB}$ wahr.

41 S. auch Pawlik (Fn. 4), S. 88 f. (Täuschung), 282 f. (Vermögensschaden).

42 Treffend Heinrich, in: Arzt/Weber/Heinrich/Hilgendorf (Fn. 3), §20 Rn. 26. Zur Entstehungsgeschichte Maurach/Schroeder/Maiwald, Strafrecht BT 1, 10. Aufl. 2009, $\$ 41$ Rn. 3 ff.

43 Zur Abgrenzung von Begriff und "contested concept" Dworkin, Bürgerrechte ernstgenommen, 1984, S. 178. S. ferner Bittner, Recht als interpretative Praxis, 1988 , S. $30 \mathrm{f}$.

44 Näher dazu v. Hippel, Rechtstheorie und Rechtsdogmatik. 1964, S. 15; Kubiciel (Fn. 35), S. 102 ff., $115 \mathrm{ff}$. Zur Bedeutung einer das System zusammenhaltenden Strafrechtswissenschaftstheorie Pawlik, Festschrift für Jakobs, 2007, S. 469 ff. 
henden Konflikte im Wege einer Ad-hoc-Argumentation aufzulösen versucht.

Angesichts dessen fragt sich, wo ein leitendes Prinzip, das einigermaßen konsensfähig sein könnte, zu finden ist. Als fruchtbar für die Neukonzeptualisierung des Vermögensbegriffs und damit für wesentliche Teile der Dogmatik der $\S \S 263,266$ StGB könnte sich der Blick auf das Zivilrecht erweisen, dessen Prägung normativer Begriffe wie Eigentum das (zumindest limitiert akzessorische) Strafrecht auch an anderen Stellen übernimmt. ${ }^{45}$ Im Zivilrecht wird als Vermögen die Gesamtheit aller Rechte, Forderungen und geldwerten Rechtsverhältnisse einer Person bezeichnet. ${ }^{46}$ Dies zeigt: Vermögen ist ein primär normatives Konzept, bei dem Geldwertes zu Rechten, Forderungen oder Rechtsverhältnissen erstarkt sein muss. Rechte, Forderungen oder Rechtsverhältnisse stehen aber einer Person zu, nicht der Rechtsgemeinschaft. Auf das Strafrecht übertragen heißt dies, dass das Strafrecht das Vermögen nicht primär als Institution schützt, sondern so, wie es das Vermögen im Zivilrecht vorfindet: als subjektives Recht. ${ }^{47}$ Da das Spezifikum der Rechtsfolge Strafe darin liegt, dass sie auf die Verletzung eines besonderen Rechts als allgemeines Recht antwortet, ${ }^{48}$ kann es Betrug weder ohne eine Verletzung eines konkreten Vermögensrechts noch ohne eine Verletzung des allgemeinen Rechts, welches die Institution Vermögen ausgestaltet, geben. Daraus lässt sich zum einen ableiten, dass sittenwidrig-nichtige Forderungen oder rechtswidrige Besitzlagen ungeachtet ihrer ökonomischen Bewertung kein strafrechtlich geschütztes Vermögen darstellen. Darüber hinaus folgt daraus, dass der

45 Näher Pawlik (Fn. 4), S. 254: Das Strafrecht dürfe die Vorgaben des Zivilrechts nicht außer Acht lassen, müsse aber einen eigenständigen, freiheitstheoretisch fundierten Vermögensbegriff verwenden. Im Grundsatz ebenso Wirtig (Fn. 1). S. 190.

46 Klunzinger, Einführung in das Bürgerliche Recht, 16. Aufl. 2013, S. 65: „Summe aller geldwerten Rechte einer Person". Ähnlich Medicus, Bürgerliches Recht Allgemeiner Teil, 10. Aufl. 2010, Rn. 1198; s. ferner Stresemann, in: Münchener Kommentar zum Bürgerlichen Gesetzbuch. Bd. 1, 6. Aufl. 2012, §90 Rn. 43: ,(..) Summe aller geldwerten Güter einer natürlichen oder juristischen Person; es besteht nur aus Rechten, dh. aus dem Eigentum an den einzelnen Gegenständen."

47 Hefendehl (in diesem Band), S. 77 ff., insbes. S. 83. Ferner Saliger (Fn. 4), § 263 Rn. 1; Seier, in: Achenbach/Ransiek (Hrsg.), Handbuch Wirtschaftsstrafrecht, 4. Aufl. 2015, S. 731. Dass der (wirtschaftliche) Vermögensbegriff der h.M. die Wertungen einer „rechtlich präformierten sozialem Welt" zu berücksichtigen hat. betont auch Saliger (Fn. 16), S. 24.

48 Umfassend dazu Pawlik (Fn. 35), S. 90 ff., 110 ff.; Kubiciel (Fn. 35), S. 159 ff. (insbes. S. $167 \mathrm{f}$.). 
konkrete Wert eines Vermögensrechtes nicht unabhängig von den Vorstellungen der Inhaber der Rechte bestimmt werden kann, so dass im Fall des Eingehungsbetruges die ,intersubjektive Wertsetzung" durch die Parteien beim Vertragsschluss „die Basis der Schadensfeststellung“ sein muss. ${ }^{49}$ Auch für das hier thematisierte Spezialproblem des Vermögensschadens bei Personengesellschaften erweist sich dieser Ansatz als fruchtbar: Wo bzw. bei wem ein Schaden eintritt, beantwortet sich nicht nach ökonomischen Maximen, sondern nach jenen des Zivilrechts als „Leitrechtsmaterie". Wenn das Vermögen aber die Gesamtheit von Rechten, Forderungen und Rechtsverhältnissen einer Person ist, und wenn das Vermögen nicht primär als Institution oder Abstraktum, sondern als Voraussetzung für die Entfaltung personaler und wirtschaftlicher Freiheit geschützt wird, ${ }^{50}$ dann kann die Vermögensfähigkeit ${ }^{51}$ der Personengesellschaft nicht ausreichen. Entscheidend ist vielmehr, dass das Recht den faktischen Träger des Vermögens als Person anerkennt und mit der Möglichkeit ausstattet, eigene Entscheidungen zu treffen (dazu III. 3.). Dies ist bei Personengesellschaften nicht bzw. in einem nicht ausreichenden Maß der Fall. Aus diesen Gründen ist der Rechtsprechung zum (fehlenden) Vermögensnachteil bei Personengesellschaften zuzustimmen.

Betrachtet man all dies zusammen, gibt es (auch in der Rechtsprechung der Strafsenate des BGH) Argumentationslinien und Gründe dafür, den Vermögens- bzw. Schadensbegriff zu renormativieren und auf dieser Basis neu zu konzeptualisieren. ${ }^{52}$ Innerhalb des $\S 263$ StGB spannte man damit zugleich den Bogen zu den eingangs (I. 1.) erwähnten Normativierungen des Täuschungsbegriffs, ${ }^{53}$ so dass der Tatbestand des Betruges insgesamt

49 So BGH JZ 2014, 96, 98. - Dass Rechtsprechung und h.L. aus dieser grundsätzlichen Einsicht bislang keine weitergehenden Konsequenzen gezogen haben, liegt an der Befürchtung, dass jede ,persőnliche Enttäuschung“ hinsichtlich des Erlangten zu einem Schaden hinaufgestuft werden könnte, so prägnant Maurach/Schroeder/Maiwald (Fn. 31), § 41 Rn. 93. Indes lässt sich dieser Gefahr begegnen, wenn man verlangt, dass der Gegenstand der persönlichen Enttäuschung einen Tauschwert in Geld haben muss.

50 Umfassend Kindhäuser (Rn. 4), §263 Rn. 35 ff.; grds. zustimmend Rönnau (in diesem Band), S. 31, 40.

51 Stölting (Fn. 17), S. 98, nach Hadding, ZGR 2001, 712, 718.

52 Năher dazu Pawlik (Fn. 4), S. 259 ff.

53 Zu einer Normativierung des Irrtumsbegriffs in Abgrenzung zum (vorgeblich) psychologischen .,sachgedankliche Mitbewusstsein“" Kubiciel, HRRS 10/2015 (im Erscheinen). 
auf einer neuen, tragfähigeren, weil systematisch kohärenten Basis stünde. Man darf gespannt sein, ob und wie weit diese - an unterschiedlichen Stellen aufscheinenden - normativierenden Ansätze gegen die zu erwartenden Widerstände der herrschenden, traditionell empirisch ausgerichteten Sichtweise vorangetrieben werden. 